\title{
THE DEVELOPMENT OF THE COLD ROLLING TEXTURE IN SOME HEXAGONAL MATERIALS : EXPERIMENTAL STUDY AND MODELIZATION
}

\author{
M.J. PHILIPPE, F. WAGNER, F.E. MELLAB and C. ESLING \\ Laboratoire de Métallurgie des Matériaux Polycristallins, \\ ISGMP - UFR MIM - Sciences \\ F - 57045 Metz Cedex 01
}

\section{Introduction}

In order to calculate mechanical properties of hexagonal materials, as yield loci or forming limit diagrams, for example, it is first necessary to understand their texture evolution during plastic deformation. This requires in particular the knowledge of the deformation mechanisms with their critical resolved shear stresses. Few data of these CRSS are avaible in the literature. Moreover the alloying of such materials can influence them significantly (1). Transmission electron microscopy is the main tool to determine the activated deformation systems and, by considering the frequency of their occurence, to address relative values to the associated CRSS.The comparison of simulated textures with experimental ones can then be used to estimate the relevance of such data if the deformation model involves assumptions which are not too far from the real behaviour of the material during plastic deformation.In the present paper information obtained by TEM for several hexagonal alloys together with the Taylor model under various conditions $(F C=$ full constrained ; $\mathrm{RC}$ relaxconstrained) according to the grain shapes are used for texture simulation. In spite of its known limitations, the Taylor model is quite interesting because it allows to take into account twinning deformation mechanisms by using some simplifying assumptions $(2,3)$ which is not possible, at the moment, with self consistent models.

Application to Zinc alloys

The first example concerns different zinc alloys. In $\mathrm{ZnCu}$ alloys with $0.2 \% \mathrm{Cu}$ there is no second phase. In $\mathrm{ZnCuTi}$ with $0.2 \% \mathrm{Cu}$ and $0.07 \% \mathrm{Ti}$ a second phase $\mathrm{TiZn} 15$ does exist.

Sheets were rolled at $0^{\circ} \mathrm{C}$ and $-50^{\circ} \mathrm{C}$. It is indeed necessary to be aware of the strong possibility of recrystallization which starts at low temperature. Recrystallized grains are for example 
systematically present in both $\mathrm{ZnCu}$ and $\mathrm{ZnCuTi}$ alloys after $50 \%$ deformation at $0^{\circ} \mathrm{C}$. The presence of the second phase in $\mathrm{ZnCuTi}$ leads to differences in grain shape distribution which is illustrated on micrographs of figure 1. Whereas in $\mathrm{ZnCuTi}$ the grains deform approximatively as the sample does, their shapes are much more inhomogeneous in $\mathrm{ZnCu}$. The $\mathrm{ZnCuTi}$ alloy exhibits a strong texture after $80 \%$ cold rolling at $-50^{\circ} \mathrm{C}$. In the (00.2) pole figure the main peak lies in the (ND, RD) plane at about $20^{\circ}$ from ND and a small one is around RD (figure 2). This small peak disappears progressively when recrystallisation occurs. The axes $\langle 10.0\rangle$ lie mainly in the vicinity of equator with a small and spread reinforcement around TD (figure 2).

$\mathrm{ZnCu}$ shows the same components but the texture is smoother which is likely induced by the heterogeneous grain shapes and a few activity of twinning.In $\mathrm{ZnCuTi}$ electron microscopy discloses basal, cross slip with a Burgers vector and pyramidal slip.

The modelization was worked out by using the Taylor FC model with these slip systems and the following relative CRSS : basal glide $=1 ;$ prism glide $=7 ;\{11.2\}$ pyramidal $\langle c+a\rangle$ glide $=7$. The initial texture was considered as random and described by a set of orientations. Figure 3 shows the pole figures (00.2) and (10.0) after simulation of plane strain deformation up to $80 \%$ reduction. As it is often the case with the Taylor model the sharpness of the calculated texture is higher as the one of the experimental texture. The spread of the axes $\langle 10.0\rangle$ is much more smaller in the calculated texture but one can recognize the main features of the experimental texture.

Application to titanium and zirconium $\alpha$ alloys

The titanium and zirconium $\alpha$ alloys show different cold rolling textures, which can be classified according to Philippe et al (4). All the textures observed range between two extreme types: type I corresponding to $\mathrm{Ti} 35$ or to $\mathrm{Zr}$ with a low level of oxygen and type III corresponding to $\mathrm{Zy} 4$ or $\mathrm{Zr}$ with a high level of oxygen.

In type III, the active deformation mechanisms as noticed in TEM do not include twinning, but if any, there could be a few twins in tension only.In type $I$, the set of active deformation mechanisms includes twinning both in tension and in compression.

The results obtained with the type III of the deformation mechanism, without compression twins, had already been shown at ICOTOM 8 (2) with the following CRSS: prism glide=1 ; pyramidal $<$ a $>$-glide $=20$; pyramidal $<c+a>$-glide $=20$ and $\{1012\}$ twins $=9$. The simulations had led to a texture with $c$ axes in RD, which corresponds well to the experimental results. 
In type I, the active mechanisms are prismatic glide, cross-slip with $\langle a\rangle$ - Burgers vector, $\{10 \overline{1} 2\}$ tension twins and $\{11 \overline{2} 2\}$ compression twins.In titanium T35 $\alpha$ alloy, twinning is active up to $50 \%$ deformation in cold rolling (fig. 4). After that stage, the shape of grain is strongly anisotropic and no further twinning could be seen, pyramidal $\langle c+a\rangle$ glide becomes active. In the latter case the experimental texture after $80 \%$ cold rolling shows $c$ axes in transverse direction and $\langle 10 \overline{10}\rangle$ in $R D$ (fig 5).At only $50 \%$ cold rolling the texture is already the same but smoother.

Based on these observations, we have made the modelisation in two stages : in the first stage from 0 to $50 \%$ deformation FC Taylor is used with the following values for the CRSS: prism glide $=1$; pyramidal a -glide $=4.5$; twinning $\{10 \overline{1} 2\}=4.5$; twinning $\{1122\}=10$. The results obtained can thus be compared with the experiment and they show a good agreement. In the second stage, from $50 \%$ to $80 \%$ deformation, starting with the texture obtained at the first stage as the initial texture, RC Taylor is used with the following values for the CRSS: prism glide $=1$; -pyramidal $<a>$-glide $=1,5$ and pyramidal $<c+a>$-glide $=10$.In the second stage too, the agreement of the pole figures and the ODF obtained (fig.6) is quite good, for both Titanium and Zirconium with the chosen deformation mechanisms and the assessed critical resolved shear stresses.

It is known that for BCC metals the FC Taylor model alone only gives a rough indication of the general shape of the texture and its major components. To obtain a better quantitative agreement with the experiment requires to resort to the $R C$ Taylor model $(5,6)$

For hexagonal materials, Taylor's model proves to work successfully in the sequence FC-model, RC-model. This sequence fits the best to the actual metallurgical state and to the evolution of this state. It is also necessary to base the modelisation on an extensive study by electron microscopy, in order to determine the activated mechanisms contributing to the plastic deformation.

CONCLUSION,

Thus, it appears that the Taylor model applied on a sequence of full constrained followed by relax-constrained models accounts for the development of rolling texture in hexagonal materials, provided the choice of eligible deformation mechanisms with their corresponding critical resolved sheer stresses is adapted to the material considered. The major components of the rolling textures are determined by the stable orientations of the flow field associated with gliding : the activation of twinning lowers the intensity of the texture but does not as such modify the major components. However as $\{1 \overline{1} 22\}$ twinning is in 
competition as a secondary accommodation mode, with the pyramidal gliding, its activation prevents that of pyramidal gliding thus modifying indirectly the rolling texture.

(1 M.J. PHILIPPE , C. ESLING and B. HOCHEID

Textures and Microstructures vol.7 (1988) 265-303

(2 G. Y CHIN, W.F. OSFORD and D.R. MENDORF

Proc. Roy. Soc. A309 (1969) 433.

(3 P. VAN HOUTTE and F. WAGNER

Preferred orientations in deformed materials : an introduc-

tion to modern texture analysis (1985) Ch.11.

(4 M.J. PHILIPPE, F. WAGNER and C. ESLING ICOTOM 8 (1987) 837.

(5 P. VAN HOUTTE ICOTOM 7 Netherlands (1984) 7

(6 F. WAGNER, G. CANOVA, P. VAN HOUTTE and A. MOLINARI

Proceedings of ICOTOM 9 (to be published)
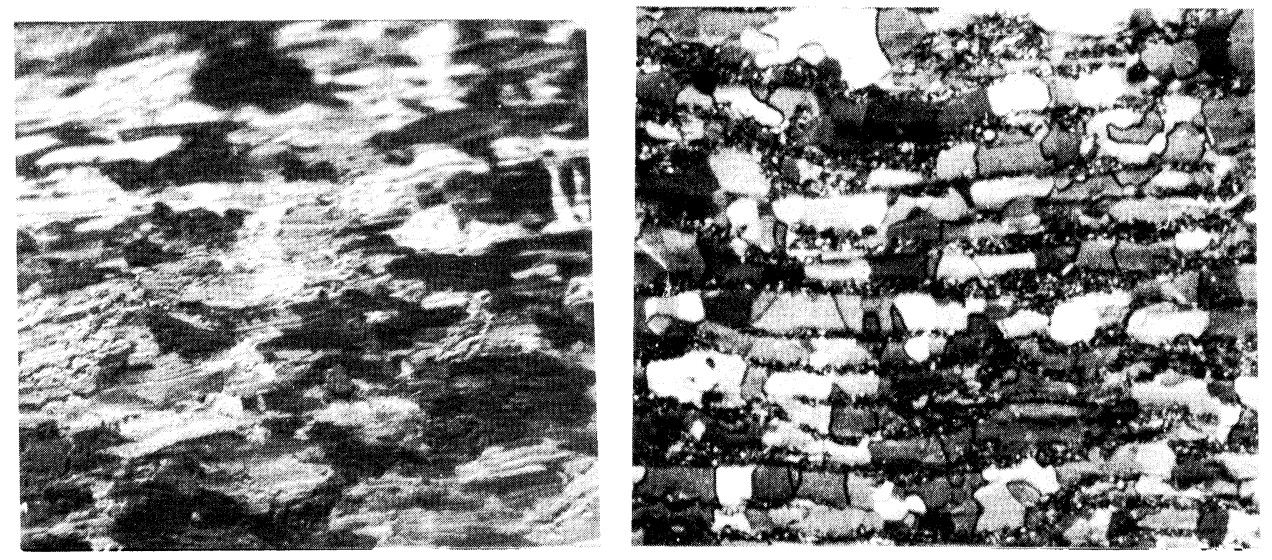

Fig. 1. Micrographs of $\mathrm{ZnCu}$ and $\mathrm{ZnCuTi}$ after $15 \%$ cold rolling

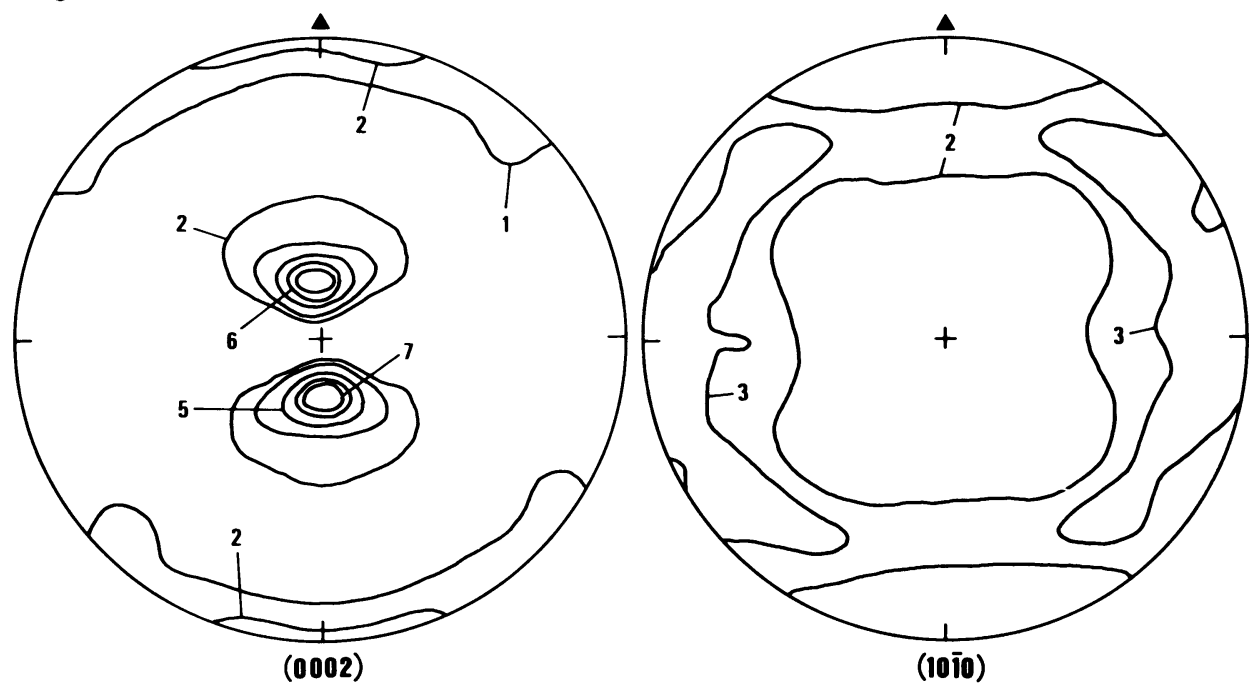

Fig. 2. Experimental pole figure for $\mathrm{Zn} \mathrm{Cu}$ Ti after $80 \%$ cold rolling. 

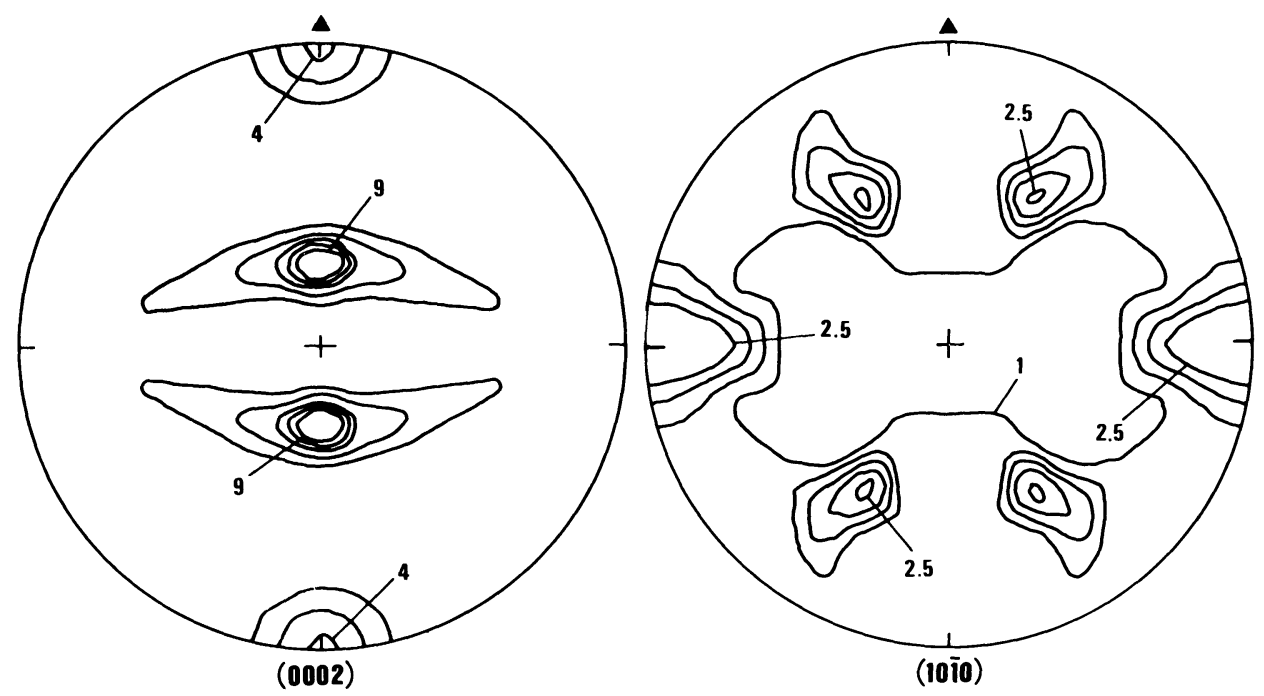

Fig. 3. Calculated polc figurc (Taylor model full constrain) after $80 \%$ cold rolling for $\mathrm{Zn} \mathrm{Cu} \mathrm{T}$.
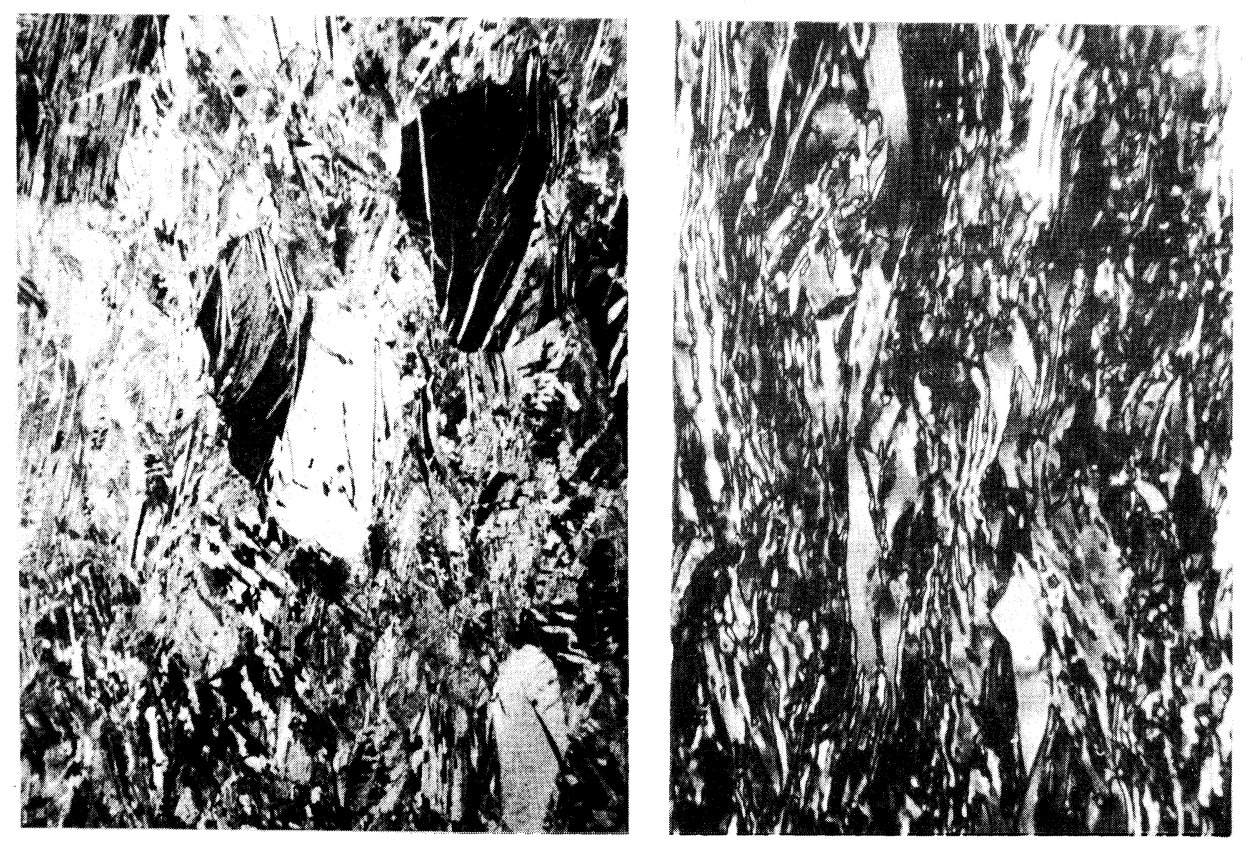

Fig. 4. Micrographs from Titanium deformed by cold rolling. 


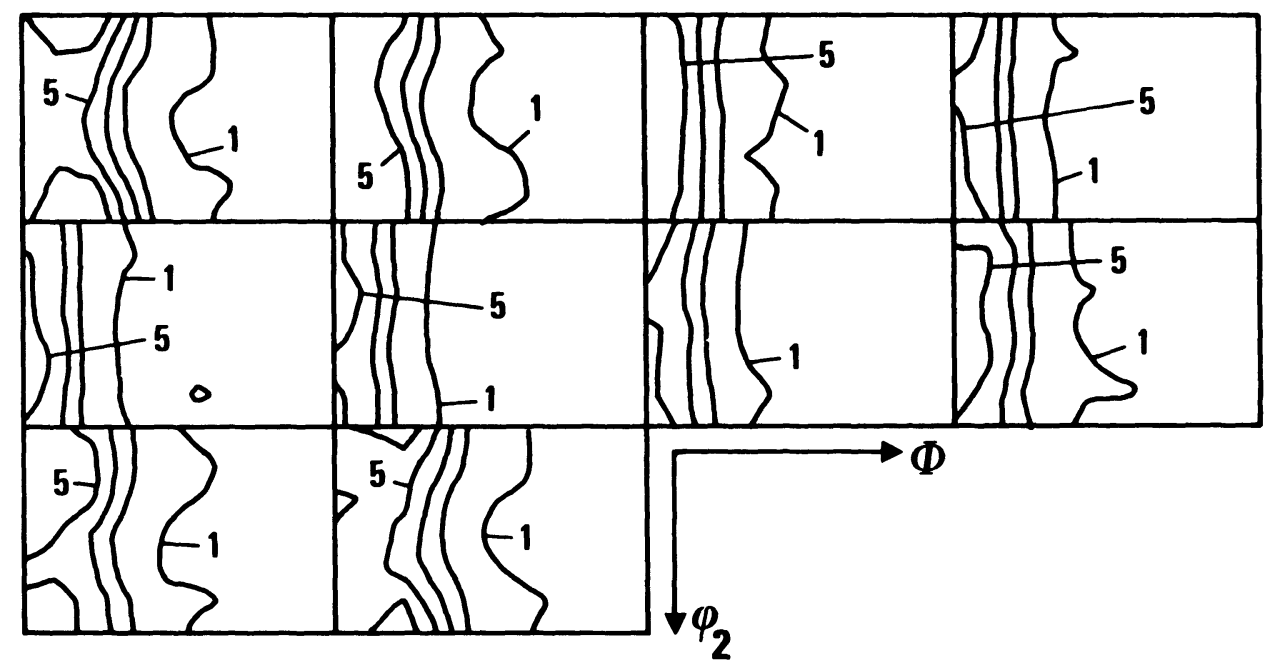
Fig. 5. Experimental F.D.O for Zirconium after $80 \%$ deforma-
tion.

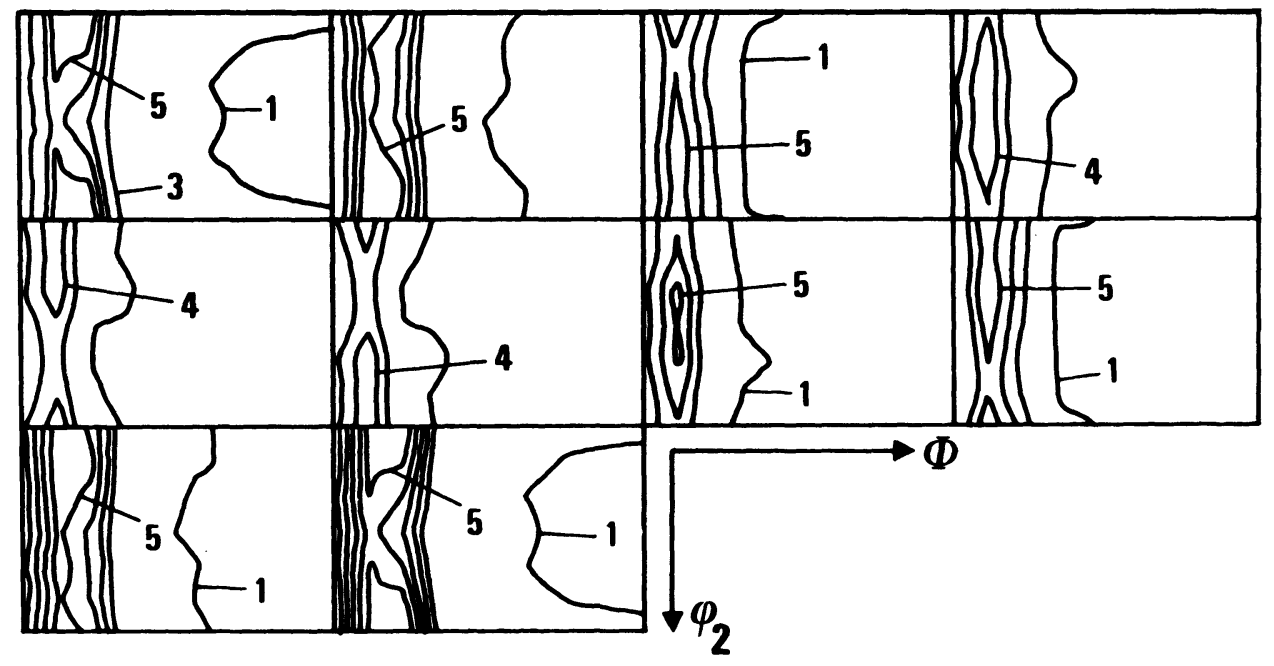

Fig. 6. Calculated F.D.O. for Zirconium $\alpha$ after $80 \%$ deformation. 